\title{
Outcomes of Corneal Topography among Progressive Keratoconus Patients 12 months following Corneal Collagen Cross-Linking
}

This article was published in the following Dove Press journal: Clinical Ophthalmology

\author{
Bader S AIQahtani ${ }^{1-3}$ \\ Saeed Alshahrani (DD ${ }^{1,2}$ \\ Waleed W Khayyat ${ }^{4,5}$ \\ Maram EA Abdalla-Elsayed ${ }^{6}$ \\ Abdullah A Altalhi ${ }^{3,4}$ \\ Amjad A Saifaldein ${ }^{7}$ \\ Mohammed A Tahal,2 \\ 'College of Medicine, King Saud Bin \\ Abdulaziz University for Health Sciences, \\ Riyadh, Saudi Arabia; ${ }^{2}$ King Abdullah \\ International Medical Research Center, \\ Riyadh, Saudi Arabia; ${ }^{3}$ Department of \\ Ophthalmology, King Abdulaziz Medical \\ City, Ministry of National Guard Health \\ Affairs, Jeddah, Saudi Arabia; ${ }^{4}$ College of \\ Medicine, King Saud Bin Abdulaziz \\ University for Health Sciences, Jeddah, \\ Saudi Arabia; ${ }^{5}$ King Khalid Eye Specialist \\ Hospital, Riyadh, Saudi Arabia; \\ ${ }^{6}$ Department of Ophthalmology, Jeddah \\ Eye Hospital, Jeddah, Saudi Arabia; \\ ${ }^{7}$ Department of Ophthalmology, College \\ of Medicine, King Saud University, Riyadh, \\ Saudi Arabia
}

Correspondence: Saeed Alshahrani College of Medicine, King Saud Bin Abdulaziz University for Health Sciences, Ar Rimayah, Riyadh II48I, Saudi Arabia Tel +966-53-089- 5550

Email saeedalshahrani158@gmail.com
Purpose: This study aimed to assess the overall and specific topographic changes among patients who underwent corneal collagen cross-linking (CXL) due to progressive keratoconus.

Methods: This retrospective case series study was conducted at a single-arm hospital in King Abdulaziz Medical City, Riyadh. All progressive keratoconus patients who underwent CXL between January 2017 and December 2018 were included consecutively. The epi-off crosslinking technique (Dresden protocol) was applied in all patients. The topographic values were measured preoperatively and 12 months postoperatively. Patients with a history of a previous corneal procedure, corneal trauma, or any corneal scarring were excluded.

Results: Among our population (29 eyes of 24 patients), 58.6\% of eyes were for male patients, and the mean age of the population was $27.76 \pm 4.21$ years. Based on the topography results, the mean values of corneal thickness at central $3 \mathrm{~mm}$ decreased from $473.45 \pm 38 \mu \mathrm{m}$ to $465.72 \pm 41.78 \mu \mathrm{m}$ following $\mathrm{CXL}(\mathrm{Z}=-1.93,95 \%$ confidence interval $[\mathrm{CI}]=0.048-0.057, \mathrm{p}=0.053)$. Clinically significant astigmatism measurements were present in 28 (96.6\%) eyes before CXL compared to 26 (89.7\%) eyes after CXL. The mean values of astigmatism among the patients were $3.37 \pm 2.25$ diopters before and $3.67 \pm 2.61$ diopters after CXL $(Z=-1696,95 \%$ confidence interval $[C I]=0.085-0.096, p=0.09)$. After CXL, the mean values of the front elevation at the apex changed from $33.90 \pm 20.13 \mu \mathrm{m}$ to $36.10 \pm$ $21.09 \mu \mathrm{m}(\mathrm{Z}=-2.792,95 \%[\mathrm{CI}]=0.003-0.006, \mathrm{p}=0.005)$. The mean values of the back elevation at the apex changed from $68.4 \pm 35.66 \mu \mathrm{m}$ to $69.90 \pm 35.89 \mu \mathrm{m}(\mathrm{Z}=-0.934,95 \%$ $\mathrm{CI}=0.343-0.366, \mathrm{p}=0.35)$.

Conclusion: The topographic corneal parameters improved significantly in the patients with corneal ectasia after CXL. These results revealed the safety and efficacy of CXL in stabilizing keratoconus progression among Saudi patients at 1 year of follow-up.

Keywords: corneal collagen cross-linking, keratoconus, topography, corneal ectasia

\section{Introduction}

Keratoconus $(\mathrm{KC})$ is a bilateral, non-inflammatory, and progressive corneal ectasia, which affects almost 1 person in every 2000. ${ }^{1}$ Aging itself induces a natural process of corneal crosslinking, which occurs through an enzymatic or non-enzymatic pattern. ${ }^{2}$ For more than a decade, corneal collagen crosslinking (CXL) has been considered the only method available to increase corneal biomechanical power and treat $\mathrm{KC}^{3}$ In CXL, riboflavin activated by ultraviolet A (UVA) light is used to increase corneal collagen fiber connections, which stabilizes the biomechanical 
measures. ${ }^{4,5} \mathrm{CXL}$ is increasingly being used as a treatment modality to halt or reduce the progression of $\mathrm{KC}$. Furthermore, CXL is thought to reduce the steepness of the cone and improve uncorrected visual acuity, corrected distance visual acuity, and subjective visual symptoms to a certain extent. ${ }^{6}$

Wollensek described the standard CXL protocol, which requires debridement of the corneal epithelium around the central 7-9 $\mathrm{mm}$ diameter and a 30-minute presoak with riboflavin 5-phosphate drops in a $20 \%$ dextran solution. The corneal tissue is then exposed to UVA rays with a 365 $\mathrm{nm}$ wavelength for 30 minutes. This process enhances the corneal tensile strength and prevents deformation or thinning of the corneal profile. ${ }^{4}$ Complications of this procedure may include postoperative pain, infectious infiltrates, sterile corneal opacity, or improper healing of the corneal epithelium. Several modifications to the riboflavincontaining solution, duration of UVA wave exposure, and irradiation techniques have been observed in transepithelial and accelerated protocols. ${ }^{7}$

The prevalence of corneal ectasia is relatively higher among Arabs and people of Eastern Mediterranean descent. ${ }^{8,9}$ A population-based study conducted in Saudi Arabia revealed an incidence of $20 \mathrm{KC}$ cases per $100,000{ }^{10}$ Variations in $\mathrm{KC}$ epidemiology may be attributed to genetic, environmental, and age-related factors. ${ }^{8}$ For example, Assiri et al found that $16 \%$ of patients with $\mathrm{KC}$ had a positive family history of corneal ectasia. ${ }^{10}$ Young people are also expected to have a higher prevalence of $\mathrm{KC}$ compared to other age groups., ${ }^{9,11}$ In Egypt, Sidky et al reported that $50.8 \%$ of children affected with subclinical $\mathrm{KC}$ were between 6 and 12 years of age, and 49.2\% were between 12 and 18 years. ${ }^{9}$ A multicenter study conducted in 2020 in Riyadh estimated a $4.79 \%$ prevalence among the pediatric population aged $6-21$ years. ${ }^{11}$

The published literature demonstrates good results after CXL for $\mathrm{KC}$. The main reported outcomes usually include uncorrected visual acuity, best-corrected visual acuity, refraction, and corneal thickness at baseline and at regular time intervals after CXL. Several studies have discussed the effect of corneal topographical changes following CXL. ${ }^{12-17}$ This includes reductions in central corneal thickness measurements, maximum keratometric values, and degrees of astigmatism. A transient increase in elevation maps values was reported within the first year following CXL, but eventual reduction of their values and achieving corneal stability occurred in studies with longer follow-up period. ${ }^{14,15}$ However, to the best of our knowledge, the corneal topography parameters pre- and post-CXL at specified time intervals have not been quantitated extensively in Saudi population. Additionally, the role of ethnic background in cornea behavior following CXL is not yet understood. We believe that this quantification will help explain more precisely to Arab patients the most likely postoperative outcomes of CXL for $\mathrm{KC}$ over the long term. This study therefore aimed to identify the overall and specific topographic changes among patients who underwent CXL at King Abdulaziz Medical City in Riyadh, Saudi Arabia.

\section{Methods}

\section{Study Participants}

This retrospective single-arm hospital-based cohort study was conducted at King Abdul-Aziz Medical City, Riyadh. All patients (29 eyes of 24 patients) diagnosed with progressive KC who underwent CXL between January 2017 and December 2018 were included consecutively.

\section{Criteria for Diagnosis and Progression}

The diagnosis of $\mathrm{KC}$ was established clinically and based on corneal topography. $\mathrm{KC}$ was considered progressive if either the maximum keratometry (K-max), the difference between the maximum and minimum keratometry, or the corneal apex power had increased by $\geq 1$ (diopters) $\mathrm{D}$, the mean keratometry value had increased by $>0.75 \mathrm{D}$, or the central corneal thickness had decreased by $2 \%$, within 2 years of follow up. ${ }^{18}$ The Amsler-Krumeich grading system was applied to grade the cases involved in this study. Patients with grades 1, 2, and 3 were included in the study. A case was considered grade 1 if any of the following findings were present: eccentric steepening, myopia, and/or astigmatism of $\leq 5.00 \mathrm{D}$, keratometric $(\mathrm{K})$ reading $\leq 48.00 \mathrm{D}$, or Vogt's lines. Grade 2 was confirmed in eyes with myopia and/or astigmatism $>5.00 \mathrm{D}$ to $\leq 8.00 \mathrm{D}$ and a $\mathrm{K}$ reading of $\leq 53.00$ D. Patients were considered Grade 3 if myopia and/or astigmatism were between 8 and $10 \mathrm{D}$, or the $\mathrm{K}$ reading was $>53.00$ D. Patients were excluded if the corneal thickness was less than $400 \mu \mathrm{m}$, or they had central corneal scarring or corneal trauma. Those with a history of previous herpetic infection, previous corneal procedure(s), or advanced dry eye disease were also excluded.

\section{Crosslinking Procedure}

The epi-off cross-linking technique (Dresden protocol) was used in all the patients. After the central $8-10 \mathrm{~mm}$ 
corneal epithelium was removed, a riboflavin solution (0.1\% riboflavin 5-phosphate and 20\% dextran T-500) was applied every 2 minutes for 30 minutes. The cornea was then irradiated with UVA $3 \mathrm{~mW} / \mathrm{cm}^{2}$ for 30 minutes for a total of $5.4 \mathrm{~J} / \mathrm{cm}^{2}$.

\section{Main Measurements}

The topographic values were measured preoperatively and 12 months postoperatively. Pentacam ${ }^{\circledR}$ (OCULUS, Wetzlar, Germany) was used to assess the corneal topographic parameters, including thickness, degree of astigmatism in D, Maximum keratometry (K-max), and front and back elevation at the apex $(\mu \mathrm{m})$. In line with the Sydney Myopia Study and the Collaborative Longitudinal Evaluation of Ethnicity and Refractive Error Study, a cylinder value of more than $1 \mathrm{D}$ was considered clinically significant astigmatism. The front and back elevation at the apex were considered high risk if the values exceeded 12 and $15 \mu \mathrm{m}$, respectively. An inferior minus superior value (I-S value) was defined as the difference in diopters between the 2 opposing points in the vertical meridian at the central $3 \mathrm{~mm}$ from the corneal vertex. The corneal thickness values were classified into 3 categories: normal, borderline, and thin. The reference values of these categories were defined based on the average Pentacam values of the corneal topography according to the Cataract \& Refractive Surgery Today Europe. ${ }^{19}$ Corneal thickness was considered normal if the reported values were more than $500 \mu \mathrm{m}$, borderline if the values were between 470 and $500 \mu \mathrm{m}$, and thin if the values were less than $470 \mu \mathrm{m} .{ }^{19}$ The study variables were collected and coded from electronic data sources.

\section{Statistical Analysis}

The data variables were managed and analyzed using IBM SPSS software (version 22.0; Armonk, NY). The continuous variables (ie, age and the refractive and topographic parameters) were expressed as means, medians, ranges, and standard deviations (SD). The categorical variables (ie, gender and the categories of topography) were presented as frequencies and percentages (\%). The Wilcoxon signed-rank test was applied to compare the pre- and postoperative topographic outcomes. Moreover, cases were grouped based on $\mathrm{KC}$ severity into two groups namely: mild stage which includes grade 1 and 2, compared to advanced stage which included grade 3 . The overall corneal topographical responses following CXL were compared between mild and advanced $\mathrm{KC}$ stages using multivariable logistic regression analysis. The difference between postoperative and preoperative topography parameters represented the CXL effect, which is considered the input of regression analysis model in our study. A p-value $<0.05$ was considered statistically significant. Our study protocol was adhered to the principles of Helsinki. Confidentiality and anonymity were maintained by coding the patients' medical record number on a separate coding sheet, and data will not be shared. All the research team members ensured that the data remained secure and out of unauthorized reach. Ethical approval was obtained from the Institutional Board Review at the King Abdullah International Medical Research Center where patient informed consents were waived due to the retrospective nature of the study.

\section{Results}

Among our population (29 eyes of 24 patients), 17 eyes (58.6\%) were of male patients, and the mean age of the population was $27.76 \pm 4.21$ years. The mean values of myopia and degree of astigmatism were $2.2 \pm 2.52 \mu \mathrm{m}$ and $3.16 \pm 1.95 \mu \mathrm{m}$, respectively. According to the AmslerKrumeich classification, $34.48 \%, 27.59 \%$, and $37.93 \%$ of the cases were grade 1, grade 2, and grade 3, respectively (Table 1).

Before the crosslinking procedure, 6 (20.7\%) eyes had normal corneal thickness. After CXL, this number increased to $26.7 \%$. Corneal thinning was present among $19(65.5 \%)$ eyes before the procedure and decreased to 16 (55.2\%) after the procedure. Four (13.8\%) eyes had borderline corneal thinning before the procedure, and this increased to $5(17.2 \%)$ eyes after the procedure. Clinically significant astigmatism measurements were present in 28 (96.6\%) eyes before CXL compared to 26 (89.7\%) eyes after CXL (Table 2).

Based on the topography results (Table 3), the mean values of corneal thickness measurements at central $3 \mathrm{~mm}$ decreased from $473.45 \pm 38 \mu \mathrm{m}$ to $465.72 \pm 41.78 \mu \mathrm{m}$ post-CXL $(\mathrm{Z}=-1.93,95 \%$ confidence interval $[\mathrm{CI}]=$ $0.048-0.057, p=0.053)$. The mean values in diopters of astigmatism among the patients were $3.37 \pm 2.25$ before and $3.67 \pm 2.61$ after CXL. The mean values of $\mathrm{K}$-max in diopters were $55.53 \pm 7.99$ before CXL but $55.43 \pm 8.43$ after the procedure $(\mathrm{Z}=-0.529,95 \% \mathrm{CI}=0.599-0.619$, $\mathrm{p}=0.597)$. The mean I-S value decreased from $6.51 \pm$ 6.22 to $6.134 \pm 6.15 \mathrm{D}$ after the procedure $(\mathrm{Z}=-1.146$, $95 \% \mathrm{CI}=0.250-0.267, \mathrm{p}=0.252$ ). After CXL, the mean values of the front elevation at the apex changed from 
Table I Demographical Characteristics of Cases

\begin{tabular}{|c|c|c|}
\hline \multicolumn{2}{|c|}{ Variables } & \multirow{3}{*}{$\begin{array}{l}\text { Frequency(\%)/Mean (SD) } \\
12(41.4 \%) \\
17(58.6 \%)\end{array}$} \\
\hline Gender & Female & \\
\hline & Male & \\
\hline \multicolumn{2}{|l|}{ Age } & $27.76(4.206)$ \\
\hline \multicolumn{2}{|c|}{ Degree of myopia } & $2.24(2.515)$ \\
\hline \multicolumn{2}{|c|}{ Degree of manifest astigmatism } & $3.16(1.95)$ \\
\hline \multicolumn{2}{|c|}{ Grade I Amsler-Krumeich classification } & $10(34.48 \%)$ \\
\hline \multicolumn{2}{|c|}{ Grade 2 Amsler-Krumeich classification } & 8 (27.59\%) \\
\hline \multicolumn{2}{|c|}{ Grade 3 Amsler-Krumeich classification } & II (37.93\%) \\
\hline
\end{tabular}

$33.90 \pm 20.13 \mu \mathrm{m}$ to $36.10 \pm 21.09 \mu \mathrm{m}(\mathrm{Z}=-2.792,95 \%$ $\mathrm{CI}=0.003-0.006, \mathrm{p}=0.005)$. The median values of the back elevation at the apex remained the same at $67 \mu \mathrm{m}$ $(\mathrm{IQR}=47)$ before and after $\mathrm{CXL}$, while the mean values changed from $68.48 \pm 35.66 \mu \mathrm{m}$ to $69.90 \pm 35.89 \mu \mathrm{m}(\mathrm{Z}=$ $-0.934,95 \% \mathrm{CI}=0.343-0.366, \mathrm{p}=0.35$ ) (Table 3 ).

According to the multivariable logistic regression analysis (Figure 1), CXL effect on front elevation map at apex values was significantly associated with a pronounced

Table 2 Prevalence of Topographical Characteristics

\begin{tabular}{|l|l|l|}
\hline Variables & Pre-CXL & Post-CXL \\
\hline Normal corneal thickness & $6(20.7 \%)$ & $8(27.6 \%)$ \\
Borderline corneal thickness & $4(13.8 \%)$ & $5(17.2 \%)$ \\
Thin cornea & $19(65.5 \%)$ & $16(55.2 \%)$ \\
Clinically significant astigmatism & $28(96.6 \%)$ & $26(89.7 \%)$ \\
High risk front elevation in apex & $28(96.6 \%)$ & $28(96.6 \%)$ \\
High risk back elevation in apex & $23(79.3 \%)$ & $23(79.3 \%)$ \\
\hline
\end{tabular}

change in advanced $\mathrm{KC}$ compared to milder stage of $\mathrm{KC}$ $(\mathrm{OR}=2.147,95 \% \mathrm{CI}=1.106-4.167, \mathrm{p}=0.024)$.

\section{Discussion}

Since responses to CXL treatment can differ based on ethnicity, we investigated the topographic corneal changes in a cohort of Saudi patients with progressive KC in Riyadh 1 year after CXL. Our study aimed to delineate the topographic changes caused by CXL in the Saudi cohort as this ethnic group had surprisingly not previously been studied in such a manner. $\mathrm{KC}$ is the main cause of keratoplasties in the Kingdom at $40.2 \%{ }^{20}$ Additionally, the prevalence of $\mathrm{KC}$ among pediatric patients in the Kingdom is known to be considerably higher than that in other countries. ${ }^{11}$

The fact that CXL increases the intermolecular bonds in the cornea is well known; it mimics the natural crosslinking that occurs with age and in people with diabetes via advanced glycation end products. The photochemical

Table 3 Wilcoxon Signed Rank Test Applied for Topography Parameters Before and After CXL

\begin{tabular}{|c|c|c|c|c|c|c|c|}
\hline $\begin{array}{l}\text { Topographical } \\
\text { Parameters }\end{array}$ & $\begin{array}{l}\text { Pre-CXL Mean } \\
\text { Value (SD) }\end{array}$ & $\begin{array}{l}\text { Post-CXL Mean } \\
\text { Value (SD) }\end{array}$ & $\begin{array}{l}\text { Pre-CXL Median } \\
\text { Value (Range) }\end{array}$ & $\begin{array}{l}\text { Post-CXL Median } \\
\text { Value (Range) }\end{array}$ & $\mathbf{z}$ & $\begin{array}{l}\text { Lower- } \\
\text { Upper 95\% } \\
\text { Cl }\end{array}$ & P-Value \\
\hline Cornea thickness & $473.45(38)$ & $465.72(41.78)$ & $463(142)$ & 47I (152) & -1.936 & $0.048-0.057$ & 0.053 \\
\hline $\begin{array}{l}\text { Degree of topographic } \\
\text { astigmatism (D) }\end{array}$ & $3.37(2.25)$ & $3.67(2.61)$ & $2.5(9.6)$ & $3(11.6)$ & -1.696 & $0.085-0.096$ & 0.09 \\
\hline $\begin{array}{l}\text { Maximum keratometry } \\
(\mathrm{K}-\max )(\mathrm{D})\end{array}$ & $55.53(7.99)$ & $55.43(8.43)$ & $54.3(27.9)$ & $51.8(30.1)$ & -0.529 & $0.599-0.619$ & 0.597 \\
\hline $\begin{array}{l}\text { Inferior minus superior } \\
\text { value (I-S value) }\end{array}$ & $6.51(6.22)$ & $6.134(6.15)$ & $4.5(23.4)$ & $3.7(24.1)$ & -1.146 & $0.250-0.267$ & 0.252 \\
\hline $\begin{array}{l}\text { Front elevation in apex } \\
(\mu \mathrm{m})\end{array}$ & $33.90(20.13)$ & $36.10(21.09)$ & $33(94)$ & $38(100)$ & -2.792 & $0.003-0.006$ & 0.005 \\
\hline $\begin{array}{l}\text { Back elevation in apex } \\
(\mu \mathrm{m})\end{array}$ & $68.48(35.66)$ & $69.90(35.89)$ & $67(161)$ & $67(166)$ & -0.934 & $0.343-0.366$ & 0.35 \\
\hline
\end{tabular}




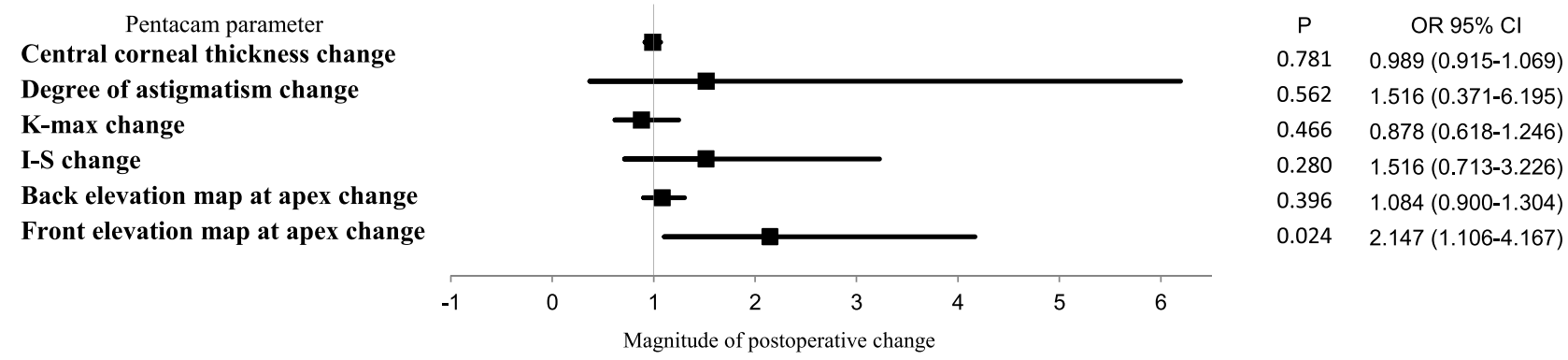

Figure I Forrest blot demonstrates a multivariable logistic regression model for postoperative changes in corneal topography parameters for advanced stage KC compared to mild stage of KC.

reaction between riboflavin and UVA is believed to cause the release of reactive oxygen species, which results in the subsequent ionization of amino groups. The effect of CXL on the anterior $250-300 \mu \mathrm{m}$ of the stroma is evident from histology, confocal microscopy, and anterior segment optical coherence tomography. ${ }^{7,21}$

Similar to reports in the literature, the use of riboflavin during the CXL procedure seemed to be effective and safe and helped reduce the progression of $\mathrm{KC}$ in our patients. ${ }^{12-17}$ For example, our study showed significant topographic changes with respect to corneal thickness and front elevation at the apex. According to the topography results, the corneal thickness decreased from $473.45 \pm 38$ $\mu \mathrm{m}$ to $465.72 \pm 41.78 \mu \mathrm{m}$ post-CXL $(\mathrm{Z}=-1.93, \mathrm{p}=$ 0.053). After CXL, the mean values of the front elevation at the apex changed from $33.90 \pm 20.13 \mu \mathrm{m}$ to $36.10 \pm$ $21.09 \mu \mathrm{m}(-2.792, \mathrm{p}=0.005)$.

However, our study did not reveal significant changes in the degrees of astigmatism $(\mathrm{p}=0.09), \mathrm{K}-\max (\mathrm{p}=$ $0.597)$, I-S values $(p=0.252)$, or back elevation at the apex $(\mathrm{p}=0.35)$. Koller et $\mathrm{al}^{22}$ investigated 7 Pentacam indices at 1 year after CXL. Four of those parameters, including the central $\mathrm{KC}$ index, index of height asymmetry, minimum radius of curvature, and $\mathrm{KC}$ index improved significantly at the 1 -year follow-up. Sadoughi et al ${ }^{14}$ found no significant differences in the pachymetric and elevation data postoperatively. The Pentacam elevation maps in our study also revealed no significant changes in the values of the anterior or posterior corneal elevation at the apex after CXL. These results are comparable to those reported by Grewal et al, ${ }^{12}$ who did not observe any significant differences in best-corrected visual acuity, spherical equivalent, cylinder vector, central corneal thickness, anterior corneal curvature, posterior corneal curvature, and posterior corneal elevation among the eyes in their study after CXL.
Anterior and posterior elevation map values are thought to be able to detect the earliest and most sensitive signs of $\mathrm{KC} ;{ }^{13,23}$ however, several studies have reported no significant changes in corneal elevation surfaces at 1 year following CXL. ${ }^{12,14-17,24}$ This difference could be explained by the fact that corneal postoperative dynamic changes may take longer than 1 year to appear. Hashemi et al discussed how corneal postoperative changes could behave differently at certain follow-up periods. ${ }^{25}$ They described an increase in corneal elevation values at the apex, which reached maximum values at 12 months postoperatively followed by a gradual reduction, with the minimum values being attained at 60 months postoperatively. ${ }^{25}$ Our findings were in line with those of this Eastern Mediterranean study, which reported significant changes in the front corneal elevation values at the apex 12 months postoperatively using Pentacam parameters; however, no significant changes were noted in the back corneal elevation values at the apex.

It is postulated that CXL induces a higher degree of corneal flattening in advanced $\mathrm{KC}$ compared to mild to moderate cases. ${ }^{26-28}$ Chan et al showed that K-max, pachymetry, and elevation map parameters were significantly affected by CXL in advanced stages of KC 12 months following accelerated CXL. ${ }^{26}$ Our cases showed similar behavior following epi-off crosslinking, and the postoperative changes were statistically significant in anterior elevations maps at apex (Figure 1). CXL is thought to penetrate relatively deeper into thinner corneas compared to less advanced thinning; hence, more dynamic postoperative corneal changes are expected. ${ }^{28}$ Corneal topographical parameters had variable responses 12 months following CXL when $\mathrm{KC}$ cases were grouped into mild stage (grade 1and2) compared to the advanced stage (grade 3). According to the multivariable logistic regression analysis, the effect of CXL on front and back 
elevation map values in apex increased more in advanced stage, particularly grade 3 , of keratoconus. However, this effect was only statistically significant in front elevations values in stage 3 of KC (Figure 1). In our study, front elevations map values could be a sensitive predictor of $\mathrm{KC}$ progression, severity, and postoperative anatomical recovery of corneal ectasia.

Since KC parameters may change based on ethnic background, ${ }^{29}$ further local studies are recommended to confirm whether corneal topographic parameters react similarly to CXL in certain populations while considering the effects of $\mathrm{KC}$ severity, baseline clinical features, and preoperative topographic indicators. The inconsistency in the results of previous studies could also be attributed to the heterogeneous nature of the $\mathrm{KC}$ cases assessed and the selection of different topographic instruments. ${ }^{30}$ Technically, Orbscan II generates the posterior corneal surface digitally, but it could miss $4 \%$ of $\mathrm{KC}$ cases and $42 \%$ of subclinical KC cases compared to the Pentacam rotating Scheimpflug camera ${ }^{30}$ used in our study.

Using the Pentacam topographer, Greenstein et al, ${ }^{31}$ reported a significant reduction in the index of surface variance, index of vertical asymmetry, $\mathrm{KC}$ index, and minimum radius of curvature 1 year after CXL therapy. However, the changes in the central $\mathrm{KC}$ index, index of height asymmetry, and index of height decentration were not statistically significant. These results provide additional evidence for the stability of the corneal surface. Even though the cornea shows an evolution toward a more regular shape (as indicated by the significant improvements in certain topographic parameters) after CXL, these may not always be in the same indices in different study populations. It is unclear why this may be. This corneal regularization is dynamic during the first year after treatment and may continue thereafter. Longer follow-up is therefore warranted to estimate the full amount of keratectasia regression after CXL in the Saudi population.

No complications were experienced in this cohort. However, several studies have reported complications ranging from insignificant stromal haze to sight-threatening infectious keratitis and sterile corneal melting. Keratitis following CXL has been reported due to herpes simplex, ${ }^{32}$ acanthamoeba, ${ }^{33}$ and a multitude of other bacteria, such as Escherichia coli and the staphylococcus and streptococcus species. ${ }^{34}$ Our study was limited by the short-term follow-up and relatively small number of patients. Additionally, bilateral involvement of $\mathrm{KC}$ in five of our patients could be a source of statistical bias. Further studies should apply more topographical variables using more advanced grading system such as Belin ABCD grading system to classify spectrum of $\mathrm{KC}$ severity as Amsler-Krumich grading system is relatively confusing and might not convey precise clinical description especially for mild and most advanced stages of the disease.

In our study, the topographic corneal parameters improved significantly in the patients with corneal ectasia following CXL. These results revealed the safety and efficacy of CXL in stabilizing $\mathrm{KC}$ progression among Saudi patients at 1 year of follow-up. However, more clinical trials with longer follow-up periods in the Saudi population are necessary to elucidate the most important corneal indices stratified according to the baseline severity of the disease to monitor the short- and long-term surgical outcomes.

\section{Ethical Approval}

All procedures performed in studies involving human participants were in accordance with the ethical standards of the institutional and national research committee and with the 1964 Helsinki declaration and its later amendments or comparable ethical standards.

\section{Disclosure}

All authors disclose that there is no conflict of interest. The article has not been presented in a meeting. The authors did not receive any financial support from any public or private sources. The authors have no financial or proprietary interest in a product, method, or material described herein.

\section{References}

1. Hofstetter HW. A keratoscopic survey of 13,395 eyes. Am J Optom Arch Am Acad Optom. 1959;36:3-11. doi:10.1097/00006324195901000-00002

2. Zhang X, Tao XC, Zhang J, et al. A review of collagen cross-linking in cornea and sclera. J Ophthalmol. 2015;2015:12. doi:10.1155/2015/ 289467

3. Ivarsen A, Hjortdal J. Collagen cross-linking for advanced progressive keratoconus. Cornea. 2013;32(7):903-906. doi:10.1097/ ICO.0b013e31828321dd

4. Wollensak G, Spoerl E, Seiler T. Riboflavin/ultraviolet-a-induced collagen crosslinking for the treatment of keratoconus. Am J Ophthalmol. 2003;135(5):620-627. doi:10.1016/S0002-9394(02)02220-1

5. Chunyu T, Xiujun P, Zhengjun F, Xia Z, Feihu Z. Corneal collagen cross-linking in keratoconus: A systematic review and meta-analysis. Sci Rep. 2014;4(10):5652-5660. doi:10.1038/srep05652

6. Corneal O' BD. Collagen cross-linking for corneal Ectasias. In: Alió JL, editor. Keratoconus: Recent Advances in Diagnosis and Treatment. Cham: Springer International Publishing; 2017:219-238. 
7. Smolek MK, Beekhuis WH. Collagen fibril orientation in the human corneal stroma and its implications in keratoconus. Invest Ophthalmol Vis Sci. 1997;38(7):1289-1290.

8. Kok YO, Tan GF, Loon SC. Review: keratoconus in Asia. Cornea. 2012;31(5):581-593. doi:10.1097/ICO.0b013e31820cd61d

9. Sidky MK, Hassanein DH, Eissa SA, Salah YM, Lotfy NM. Prevalence of subclinical keratoconus among pediatric egyptian population with astigmatism. Clin Ophthalmol. 2020;14:905-913. doi:10.2147/OPTH.S245492

10. Assiri AA, Yousuf BI, Quantock AJ, et al. Incidence and severity of keratoconus in Asir province, Saudi Arabia. $\mathrm{Br} J$ Ophthalmol. 2005;89:1403-1406. doi:10.1136/bjo.2005.074955

11. Torres Netto EA, Al-Otaibi WM, Hafezi NL, et al. Prevalence of keratoconus in paediatric patients in Riyadh, Saudi Arabia. $\mathrm{Br}$ J Ophthalmol. 2018;102(10):1436-1441. doi:10.1136/bjophthalmol2017-311391

12. Grewal DS, Brar GS, Jain R, Sood V, Singla M, Grewal SP. Corneal collagen crosslinking using riboflavin and ultraviolet-A light for keratoconus: one-year analysis using Scheimpflug imaging. $J$ Cataract Refract Surg. 2009;35:425-432. doi:10.1016/j. jcrs.2008.11.046

13. Mih'altz K, Kov'acs I. "Evaluation of keratometric, pachymetric, and elevation parameters of keratoconic corneas with pentacam,". Cornea. 2009;28(9):976-980. doi:10.1097/ICO.0b013e31819e34de

14. Sadoughi MM, Feizi S, Delfazayebaher S, Baradaran-Rafii A, Einollahi B, Shahabi C. Corneal changes after collagen crosslinking for keratoconus using dual scheimpflug imaging. J Ophthalmic Vis Res. 2015;10(4):358-363. doi:10.4103/2008-322X.176894

15. Sedaghat M, Bagheri M, Ghavami S, Bamdad S. Changes in corneal topography and biomechanical properties after collagen cross linking for keratoconus: 1-year results. Middle East Afr J Ophthalmol. 2015;22:212-219. doi:10.4103/0974-9233.151877

16. Razmjoo H, Rahgozar A, Shirani K, Abtahi SH. Pentacam topographic changes after collagen cross-linking in patients with keratoconus. Adv Biomed Res. 2015;4:62. doi:10.4103/22779175.151886

17. Pjano MA, Biscevic A, Grisevic S, Gabric I, Salkica AS. Pachymetry and elevation back map changes in keratoconus patients after crosslinking procedure. Med Arch. 2020;74(2):105-108. doi:10.5455/ medarh.2020.74.105-108

18. Gore DM, Shortt AJ, Allan BD. New clinical pathways for keratoconus. Eye. 2013;27(3):329-339. doi:10.1038/eye.2012.257

19. Sinjab MM. A 12-Point Algorithm to Master Corneal Tomography. CATARACT REFRACTIVE SURGERY TODAY EUROPE. 2017.

20. Al-Towerki AE. Changing indications for corneal transplantation at the King Khaled Eye Specialist Hospital (1983-2002). Cornea. 2004;23(6):584-588. doi:10.1097/01.ico.0000121708.58571.5b

21. Wollensak G. Corneal collagen crosslinking: new horizons. Expert Rev Ophthalmol. 2010;5:201-215. doi:10.1586/eop.10.7

22. Koller T, Iseli HP, Hafezi F, Vinciguerra P, Seiler T. Scheimpflug imaging of corneas after collagen cross-linking. Cornea 2009;28:510-515. doi:10.1097/ICO.0b013e3181915943
23. Kamiya K, Ishii R, Shimizu K, Igarashi A. Evaluation of corneal elevation, pachymetry and keratometry in keratoconic eyes with respect to the stage of Amsler-Krumeich classification. British J Ophthalmol. 2014;98(4):459463. doi:10.1136/bjophthalmol-2013304132

24. Henriquez MA, Izquierdo L Jr, Bernilla C, Zakrzewski PA, Mannis M. Riboflavin/Ultraviolet a corneal collagen crosslinking for the treatment of keratoconus: visual outcomes and Scheimpflug analysis. Cornea. 2011;30:281-6. doi:10.1097/ICO.0b013e3 181 eeaea 1

25. Hashemi H, Seyedian MA, Miraftab M, Fotouhi A, Asgari S. Corneal collagen cross-linking with riboflavin and ultraviolet a irradiation for keratoconus: long-term results. Ophthalmology. 2013;120 (8):1515-1520. doi:10.1016/j.ophtha.2013.01.012

26. Chan TC, Chow VW, Jhanji V, Wong VW. Different topographic response between mild to moderate and advanced keratoconus after accelerated collagen cross-linking. Cornea. 2015;34(8):922-927. doi:10.1097/ICO.0000000000000483

27. Sloot F, Soeters N, van der Valk R, et al. Effective corneal collagen crosslinking in advanced cases of progressive keratoconus. $J$ Cataract Refract Surg. 2013;39:1141-1145. doi:10.1016/j. jcrs.2013.01.045

28. Toprak I, Yaylali V, Yildirim C. Factors affecting outcomes of corneal collagen crosslinking treatment. Eye. 2014;28:41-46. doi:10.1038/ eye.2013.224

29. Hashemi H, Beiranvand A, Khabazkhoob M, Fotouhi A. Corneal topography patterns in the Tehran eye study: warning about the high prevalence of patterns with a skewed radial axis. Middle East Afr J Ophthalmol. 2014;21(1):72-76. doi:10.4103/0974-9233.124107

30. de Sanctis U, Loiacono C, Richiardi L, Turco D, Mutani B, Grignolo FM. Sensitivity and specificity of posterior corneal elevation measured by Pentacam in discriminating keratoconus/subclinical keratoconus. Ophthalmology. 2008;115(9):1534-1539. doi:10.1016/j. ophtha.2008.02.020

31. Greenstein SA, Fry KL, Hersh PS. Corneal topography indices after corneal collagen crosslinking for keratoconus and corneal ectasia: one-year results. J Cataract Refract Surg. 2011;37:1282-1290. doi:10.1016/j.jcrs.2011.01.029

32. Kymionis GD, Portaliou DM, Bouzoukis DI, et al. Herpetic keratitis with iritis after corneal crosslinking with riboflavin and ultraviolet A for keratoconus. J Cataract Refract Surg. 2007;33:1982-1984. doi:10.1016/j.jcrs.2007.06.036

33. Rama P, Di Matteo F, Matuska S, Paganoni G, Spinelli A. Acanthamoeba keratitis with perforation after corneal crosslinking and bandage contact lens use. $J$ Cataract Refract Surg. 2009;35:788-791. doi:10.1016/j.jcrs.2008.09.035

34. Pérez-Santonja JJ, Artola A, Javaloy J, Alió JL, Abad JL. Microbial keratitis after corneal collagen crosslinking. J Cataract Refract Surg. 2009;35:1138-1140. doi:10.1016/j.jcrs.2009.01.036
Clinical Ophthalmology

\section{Publish your work in this journal}

Clinical Ophthalmology is an international, peer-reviewed journal covering all subspecialties within ophthalmology. Key topics include: Optometry; Visual science; Pharmacology and drug therapy in eye diseases; Basic Sciences; Primary and Secondary eye care; Patient Safety and Quality of Care Improvements. This journal is indexed on PubMed

Submit your manuscript here: https://www.dovepress.com/clinical-ophthalmology-journal
Central and CAS, and is the official journal of The Society of Clinical Ophthalmology (SCO). The manuscript management system is completely online and includes a very quick and fair peer-review system, which is all easy to use. Visit http://www.dovepress.com/ testimonials.php to read real quotes from published authors. 